sheep, and they suggest that a state of tissue tolerance may exist between the freemartin and her male twin.

We are indebted to Dr. T. R. R. Mann for reading and discussing the manuscript, and one of us (N. W. M.) was supported by a Science and Industry Endowment Fund administered by the Commonwealth Scientific and Industrial Research Organization of Australia.

N. W. MOORE

L. E. A. Rowson

Agricultural Research Council

Unit of Reproductive Physiology

and Biochemistry,

Huntingdon Road,

Cambridge. Oct. 11.

${ }^{1}$ Swett, W. W., Mathews, C. A., and Graves, R. R., J. Agric. Res., 61, 587 (1940)

${ }^{2}$ Lillie, F. R., Science, 48, 611 (1916).

${ }^{3}$ Owen, R. D., Science, 102, 400 (1945).

Owen, R. D., Davis, H. P., and Morgan, R. F., J. Hered., 87, 290 (1946).

5 Anderson, D., Billingham, R. E., Lampkin, G. H., and Medawar, P. B., Hered., 5, 379 (1951).

- Moore, N. W., and Rowson, L. E. A. (unpublished results).

'Ewen, A. H., and Hummason, F. A., J. Hered., 88, 149 (1947).

${ }^{8}$ Stormont, C., Weir, W. C., and Lane, L. L., Science, 118, 695 (1953)

\section{Automatic Subdivision of Associated Populations}

Consmeration has been given in the past to the factorial analysis of qualitative data ${ }^{1}$, including that particular form in which the attributes (presence-orabsence, yes-or-no) can take only the values 0 or 1 , so that the correlation matrix is obtained from a set of $2 \times 2$ contingency tables ${ }^{2}$. Little attention has been paid to the valuable information to be obtained from the subdivision of populations of this latter type. Goodall ${ }^{3}$, working with vegetation, pointed out that such populations are divisible into "homogeneous" groups, in which all associations are either indeterminate (a given attribute being 0 or 1 in all members of the group) or below a predetermined significancelevel. Unfortunately, he elected to divide by reference to a criterion which is inefficient, and which tends to produce final groups capable of ambiguous recombination.

The problem is to find the attribute which, at each stage of subdivision, is most likely to reduce the residual associations to their lowest possible level. At first sight, an attractive solution would appear to be to divide on the attribute with the highest loading, irrespective of sign, on the first principal axis of the correlation matrix. However, although finding this presents no difficulty on an electronic computer, it involves not only calculating but also storing all the elements of the matrix, thus seriously reducing the space available for the basic data (that is, the specified individuals). A similar objection applies to the corresponding element of the first centroid axis. Furthermore, it is by no means certain that the resulting division is the best for all purposes. As has been pointed out elsewhere in connexion with the ecological application of the problem", Goodall's definition of a "homogeneous group" depends ultimately on a significance test, the simplest of which for such purposes is the calculation of $\chi^{2}$ with Yates' correction. If this criterion is in fact used, a preferable solution is to divide by reference, not to the correlation matrix, but to the corresponding contingency matrix. The correlation coefficients $(r)$ can then all be taken with the positive sign, and the attribute with the highest $\Sigma|r|$ will necessarily be the attribute with the highest loading on the first centroid axis of the contingency matrix. (We prefer to use $|r|$ rather than $r^{2}$, the mean-square contingency itself, since it provides a closer analogy with factorial analysis; experience suggests that this does not discernibly reduce the efficiency of subdivision.) Since the $|r|$ values can be summed as they are obtained, it is necessary to provide only as many storage locations as there are attributes. Except for very large projects, ample space is thus available for both programme and data, and the subdivision can be made fully automatic.

We have therefore prepared, for the Ferranti 'Pegasus' computer, a programme which, from the specifications of all the individuals concerned, will carry out completely automatic subdivision into groups homogeneous at the $P=0.05$ level of significance. The highest $\chi^{2}$ encountered during the examination of any group is printed out, thus permitting cross-comparison of all intermediate groups ; and provision is made for disregarding any attributes which it is desired to exclude from the analysis. The programme exists in two alternative forms : the first will process up to 3,360 individuals specified by not more than 38 attributes, the second up to 1,680 individuals specified by not more than 76 attributes.

The resulting information may be used in several ways. Most important is the study of the structure of the homogeneous groups themselves, with particu. lar reference to the disposition of those attributes not specifically used for subdivision. Secondly, as these latter attributes are inherently likely to be of importance in any subsequent factorial analysis, the fact that they are already known may well facilitate decisions on the placing of hyperplanes; in fact, we suspect that in some cases sufficient information may be obtained from the subdivision to render the far more laborious factorial analysis unnecessary. Lastly, if, as in ecological work, the individuals are of known distribution in space, the groups can be plotted on a map.

Botany Department,

W. T. Williams

Computation Laboratory,

University of Southampton. Oct. 14.

${ }^{2}$ Burt, C., Brit. J. Psychol. (Stat. Sect.), 3, 166 (1950).

SSlater, P., J. Roy. Stat. Soc. (Supp.), , 114 (1947).

3 Goodall, D. W., Aust. J. Bot., 1, 39 (1953).

- Williams, W. T., and Lambert, J. M., J. Ecol. (in the press).

\section{Salt Excretion in Marine Birds}

IN connexion with recent research on salt excretion in marine birds ${ }^{1,2}$ the following observation which I made some twenty-five years ago may be of interest. While cruising in a yacht far from land in the northern North Sea, I often used to watch lesser black-backed gulls as they soared above the ship or were perched on the mast head. I was very puzzled to account for a regular rain of drops of water which each bird shook at intervals from the tip of its beak. This was evidently salt excretion in action, and I know of no similar published account.

The Laboratory, Citadel Hill,

\section{F. S. RUSSELI} Plymouth.

' Schmidt-Nielsen, Knut, and Sladen, W. J. L., Nature, 181, 1217 (1958).

"Schmidt-Nielsen, Knut, and Fange, Ragnar, Auk, 75, 282 (1958). 